\title{
Despertar la reflexión en la formación docente: dispositivos efectivos
}

\section{Awakening reflection in teacher training: effective devices}

\author{
Rebeca Anijovich ${ }^{1}$, Graciela Cappelletti ${ }^{2}$ \\ ${ }^{1}$ Universidad de San Andrés, Argentina (ranijovich@udesa.edu.ar) \\ ${ }^{2}$ Universidad de San Andrés, Argentina (gachycappelletti@gmail.com)
}

\section{RESUMEN:}

Los objetivos enunciados en esta investigación, realizada en la Facultad de Derecho de la Universidad de Buenos Aires, fueron dos:

1) Relevar y sistematizar los incidentes críticos (IC) planteados por los estudiantes que cursan el Profesorado en Ciencias Jurídicas con el objeto de generar conocimientos acerca de los contenidos de las reflexiones que se producen a partir de ello.

2) Proponer estrategias de análisis de los IC que contribuyan al desarrollo de procesos reflexivos de los estudiantes del profesorado, en correspondencia con los avances teóricos internacionales en este sentido.

La metodología utilizada es de tipo cualitativo, centrada en el análisis de las reflexiones de los futuros docentes acerca de sus prácticas a través de dispositivos de trabajo dentro de un programa de formación docente.

En este estudio se analizaron 30 IC que constituyen las unidades de análisis. El material recogido pertenece a los abogados que cursaron Residencia Docente durante el 2011 y 2013 en el Profesorado de Ciencias Jurídicas de la Facultad de Derecho de la Universidad de Buenos Aires.

El número de casos estudiados no tuvo como intencionalidad la generalización sino la comprensión de cuestiones vinculadas con la reflexión de la propia práctica en la formación docente.
Las fuentes primarias que se utilizaron fueron las producciones escritas de los futuros docentes y las entrevistas en profundidad.

\begin{tabular}{|c|c|c|}
\hline PALABRAS & CLAVE: & INCIDENTES \\
\hline $\begin{array}{l}\text { CRÍTICOS, } \\
\text { DOCENTE. }\end{array}$ & REFLEXIÓN, & FORMACIÓN \\
\hline
\end{tabular}

ABSTRACT:

There were two objectives stated in this research carried out at the Law Faculty at Buenos Aires University:

1) Relieve and systematize the critical incidents posed by the students who study to became teachers in Legal Sciences in order to generate knowledge about the contents of the reflections that are produced from it.

2) Propose strategies for analyzing critical incidents that contribute to the development of reflective processes of the students of the teaching staff, in correspondence with the international theoretical advances in this sense.

The methodology used is qualitative, focused on the analysis of reflections of future teachers about their practices through work devices within a teacher training program.

In this study, 30 critical incidents that constitute the units of analysis were analyzed. The material collected belongs to the lawyers who studied Teaching Residence during 2011 and 2013 in the Law Faculty at Buenos Aires University. 
The number of cases studied did not have the intention of generalization rather the understanding of issues related to the reflection of practice itself in teacher training.

\section{KEYWORDS: CRITICAL INCIDENTS, REFLECTION, TRAINING TEACHERS.}

\section{INTRODUCCIÓN}

En las últimas décadas, las expresiones, profesional reflexivo, el profesor como investigador, se han convertido en lemas característicos a favor de la reforma y mejoras de la enseñanza y la formación del profesorado en todo el mundo. Estas expresiones suponen una valoración de la práctica docente, en tanto espacio de producción de saberes y el reconocimiento a los profesores como profesionales que tienen teorías implícitas y experiencias que pueden contribuir a la constitución de una base sistematizada de conocimientos sobre la enseñanza (Anijovich et al, 2009).

Estos conceptos, desarrollados alrededor de la idea de un profesional reflexivo, consideran al docente como quien debe desempeñar un rol activo en la formulación de sus objetivos y métodos de enseñanza, en contraposición al profesor que administra y ejecuta propuestas técnicas diseñadas desde el exterior de las aulas. Consideramos que el trabajo de investigación sobre los procesos de formación docente en esta área de conocimiento podrá favorecer las necesidades específicas en relación con la enseñanza de las ciencias jurídicas, que pueden colaborar en mejores aprendizajes de los futuros profesores.

Generar las condiciones para la formación de docentes reflexivos significa también reconocer que la producción del conocimiento respecto a lo que constituye una enseñanza adecuada, no es propiedad exclusiva de los centros universitarios y de investigación y desarrollo, sino también del análisis de las prácticas de enseñanza.

Para analizar estas prácticas disponemos de una variedad de dispositivos como los diarios de formación, los portafolios, el relato de incidentes críticos, entre otros. En esta investigación se ha trabajado sobre los IC.

La primera referencia al IC se atribuye a Flanagan (1954) que publicó un artículo sobre la técnica del IC. Según este autor se trata de una técnica flexible que se originó en el campo de la psicología industrial y organizacional durante la segunda guerra mundial para la selección y clasificación de la tripulación.

Según David Tripp (1993) los IC no son "cosas" que existen independientemente del observador y que esperan ser descubiertas. Son producidos por el modo en que tenemos de mirar una situación: lo crítico del incidente está dado por la interpretación del significado que un hecho tiene. Desde la perspectiva de este autor, para desarrollar un juicio profesional en la tarea de enseñar, es necesario reposicionarnos para mirar nuestro trabajo.

Ya en el campo de la enseñanza, Fernández González, Elórtegui Escartín y Medina Pérez (2003), Fernández González, Medina Pérez y Elórtegui Escartín (2003) caracterizan los IC como una estrategia estructurada en la que se presenta a los profesores situaciones de enseñanza en forma escrita para que las analicen y tomen decisiones en función de la información recibida. Estos últimos autores describen el uso de los IC como una estrategia de formación y perfeccionamiento del profesorado de Educación Secundaria.

El proceso se lleva a cabo en "situaciones de laboratorio sin riesgo y con posibilidad de repetir el análisis, lo que permite a los profesores acercarse a la realidad" (Fernández González, Elórtegui Escartín y Medina Pérez, 2003, p. 104). De este modo, el IC constituye una estrategia de enseñanza que promueve el análisis y reflexión sobre la práctica docente y un conocimiento más profundo sobre la realidad. En este contexto, el tratamiento de IC favorece un modo de trabajo donde los profesores nóveles explicitan su postura sobre aspectos recurrentes de la tarea docente.

Rosales (2000) expresa que el trabajo del profesor (o futuro profesor) en torno a IC de enseñanza es valioso porque:

a) Permite conocer cuáles son, a juicio del profesor, las situaciones que lo ponen en contacto con la realidad de la enseñanza.

b) Pone de relieve aspectos de la práctica profesional que raramente aparecen en la formación.

c) Sirve de punto de partida para la reflexión, con utilización de principios teóricos de interés pedagógico y didáctico.

d) Constituye un instrumento para la estimulación de la capacidad de decisión del profesor, especialmente cuando se presenta a modo de problemas didácticos, para los que es preciso encontrar soluciones óptimas.

Desde la perspectiva de Shulman (2002) es posible integrar la definición del IC como caso 
externo y como suceso experimentado por el propio docente. Sus aportes posibilitan comprender dos funciones del IC: 1) promover el análisis y la reflexión sobre el suceso acontecido y los propios supuestos y modos de actuar del docente que lo ha experimentado; 2) analizar y construir conocimientos sobre situaciones reales de enseñanza mediante la escritura de casos. Este proceso, según Shulman, se compone de las siguientes partes: 1. Lo que está sucediendo (identificando los hechos importantes del caso); 2. Análisis (planteando interrogantes y cuestiones, analizando los retos desde múltiples perspectivas, evaluando las soluciones propuestas en el caso); 3. Acción (proponiendo soluciones alternativas, teniendo en cuenta las ventajas y limitaciones de cada uno, y consecuencias a corto y largo plazo de cada uno) y 4. ¿De qué es este caso? (creando temas y principios generalizables). Si bien este proceso puede variar según el grupo con el que se esté trabajando lo que sí debe asegurarse quien guía este proceso es de tomarse el tiempo necesario para desarrollar una comprensión compartida de los hechos pertinentes del caso y analizar las preguntas y los problemas presentes, antes de pasar a la etapa de la acción.

Husu, Toom y Patrikainen (2008) enfatizan tres aspectos particulares: (1) el deseo del docente de examinar su propia práctica y desarrollo profesional; (2) el significado de la reflexión colegiada, en el sentido de que sea compartida con otros y (3) el período de tiempo requerido para reflexionar sobre un aspecto desde diferentes perspectivas, ampliando el marco de referencia. Estos autores deciden considerar los IC sosteniendo la idea de que no es posible reflexionar sobre todo lo que sucede en la clase y, en consecuencia, para lograr una reflexión profunda es preciso hacer foco. Asimismo, los IC condensan modos de actuar. Siguiendo esta lógica, cuando un incidente particular es elegido por el docente y es significativo para él, asumimos que aún ese pequeño incidente incluye los elementos básicos del modo de actuar y pensar personal del docente en un contexto pedagógico. Los autores proponen volver a analizar los IC varias veces para alcanzar perspectivas más amplias. Metodológicamente proponen filmar una clase y luego efectuar una entrevista al docente, a cargo de un colega o el investigador, en la que se promueve un recuerdo estimulado, con el objetivo de que pueda verbalizar los procesos de pensamiento que llevó a cabo en la clase filmada. En esta fase se indaga sobre preguntas tales como ¿qué?, ¿cómo? y ¿por qué? Luego se proponen discusiones reflexivas donde se pone el foco sobre el IC que el docente elige de la clase filmada. El objetivo es revisitar el incidente, considerar su sentido en un contexto más amplio y explorar posibilidades para cambiar las acciones concretas del profesor. El hecho de filmar el incidente, realizar una entrevista y a posteriori varias discusiones reflexivas propicia un pensamiento generado por la interacción entre colegas.

Tomando los aportes de Litwin (2008, p. 204), $\operatorname{los}$ IC

constituyen experiencias no planificadas, anticipadas ni controladas. Para transformarlas en un acontecimiento investigable debemos distanciarnos de nuestro papel de maestros o profesores, recoger información de los diferentes actores, conformar un grupo que estudie el incidente, documentarlo, realizar los registros y encontrar razones nuevas que formen parte del proceso de descubrimiento. El conocimiento obtenido será útil, no sólo para reconocer la crisis en toda su profundidad sino también para prevenir futuras situaciones indeseables.

Es relevante la intervención del tutor para ayudar a hacer visible el IC para quien no lo ve. Siguiendo a Harrison y Lee (2011) diremos que la conversación con el docente tutor facilita la reestructuración del incidente, permite pasar de una mera descripción de los hechos a una explicación de por qué sucedieron los mismos. En este sentido, el diálogo que se establece entre un docente experimentado y un docente en formación funciona a la manera de un puente en doble dirección: al futuro docente le permite realizar un proceso de pensamiento en el que se visibilizan aspectos acerca de la enseñanza (y su complejidad) que muchas veces quedan opacados por la situación de práctica iniciática.

Por su parte Griffin (2003) da cuenta de un estudio realizado en Estados Unidos, en el que se estudiaron 135 IC pertenecientes a 28 docentes en formación. El objetivo del estudio fue determinar los efectos de los IC y las actividades de enseñanza asociadas en las habilidades reflexivas de los docentes en formación. Los 135 IC fueron analizados teniendo en cuenta: los niveles de pensamiento y lenguaje reflexivo, el grado de orientación hacia el crecimiento y la indagación, y por último, los modos de pensamiento reflexivo. Los resultados de la investigación dan cuenta que el uso de IC y actividades relacionadas 
incrementan la capacidad reflexiva de los docentes en formación.

Harrison y Lee (2011) realizaron un estudio en Inglaterra cuyo objetivo fue investigar las experiencias de IC de futuros docentes. Consideran que los incidentes en una escuela se vuelven críticos cuando presentan al docente un dilema a través del cual hay por lo menos dos cursos de acción que son mutuamente excluyentes. En la formación de futuros docentes dichos incidentes permiten moverse más allá de un relato descriptivo hacia el análisis de los hechos. Entre los hallazgos encontrados se destacan que los criterios por cuales los futuros docentes eligieron los IC que elaboraron fueron por razones prácticas, técnicas o emocionales. La mitad de los incidentes estudiados se refirió al manejo de la clase y al comportamiento de los residentes. Un cuarto de las producciones se refirió a la enseñanza y el aprendizaje en clase y otros se refirieron a cuestiones ligadas a los vínculos y consejos de convivencia. Por último, se destaca cómo para los futuros docentes el acompañamiento personal y el uso de las emociones son centrales para habilitar una práctica reflexiva y para desarrollar niveles de pensamiento crítico más profundos.

La recuperación y utilización de lo aprendido en situaciones de actuación resulta crítico en el caso de la formación de los docentes, lo cual acarrea la tensión entre la teoría y la práctica que se presenta repetidamente en los debates acerca de dicha formación. Una de las tendencias es poner un mayor énfasis en la práctica como parte de la formación, pero, así como los profesionales no pueden renunciar a la intervención, tampoco pueden soslayar el aporte de un conjunto de disciplinas que sintetice el conocimiento sobre el objeto de su actuación.

Para ello resulta necesario referir al énfasis en el desarrollo de la práctica reflexiva. En el trayecto de formación docente partimos de distinguir, tal como menciona Perrenoud (2004), a la reflexión en plena acción, la reflexión fuera del impulso de la acción, la reflexión sobre el sistema de acción. A esta diversidad de los objetos y niveles de reflexión hay que añadir la de los estilos cognitivos y las situaciones concretas. No todos los sujetos reflexionan del mismo modo. Para saber cómo un practicante o un docente novel reflexionan en plena acción, sobre la acción, sobre los conocimientos y sus esquemas de acción o también sobre los sistemas de acción colectiva en los que está implicado, es necesario observarlo y, además, establecer un diálogo profundo.

\section{MATERIAL Y MÉTODO}

Se trata de una investigación de tipo cualitativo, centrada en el análisis de las reflexiones docentes sobre sus prácticas a través de dispositivos de trabajo dentro de un programa de formación docente. El fenómeno por analizar es complejo y no puede ser abordado a través de mediciones o verificaciones causa-efecto. Se trata de descubrir y analizar procesos.

La investigación se llevó a cabo dentro de los enfoques que intentan observar, comprender, confrontar práctica con teoría, efectuar asociaciones, formular preguntas no previstas y generar nuevos conocimientos.

En este estudio se analizan 30 IC que constituyen las unidades de análisis. El material recogido pertenece a los residentes que cursaron Residencia Docente durante el 2011 a 2013 en el Profesorado de Ciencias Jurídicas de la Facultad de Derecho de la Universidad de Buenos Aires.

El número de casos estudiados no tiene como intencionalidad la generalización, sino la comprensión de cuestiones vinculadas con la reflexión de la propia práctica en la formación docente.

Las fuentes primarias que se utilizaron fueron las producciones escritas de los estudiantes y las entrevistas en profundidad.

El trabajo con producciones escritas de los estudiantes como fuente para la investigación presenta ventajas y limitaciones. En cuanto a las primeras, reconocemos al proceso de escritura como una práctica activa y personal, constituye un testimonio directo y refiere a múltiples representaciones. Las limitaciones refieren a que los textos pueden ser "escritos" para el docente del curso, un "deber ser" más artificial que una construcción genuina.

La consigna planteada a los futuros docentes para que evoquen y registren un IC fue la siguiente: relatar algún suceso vivido en su actividad de Residente Docente que le parezca que se enmarca en la definición que encontrará en este texto. Considere si pudo resolverlo, ¿cómo lo resolvió? y si tuvo impacto en su desempeño futuro.

La definición aportada fue: "un suceso acotado en el tiempo y en el espacio que desestabiliza los modos habituales de actuar del profesor y pone en crisis (en mayor o menor medida) su accionar. Es decir, el profesor entiende que 'debe hacer algo' a partir de esa vivencia".

El recorrido de la práctica a la teoría fue realizado en varias oportunidades a lo largo del 
trabajo y supuso, por ende, un proceso de retroalimentación mutua.

Luego, el trabajo en terreno permitió contrastar permanentemente con los conceptos teóricos para promover un enriquecimiento de estos $\mathrm{y}$, en ese proceso, volver a las preguntas iniciales.

Como toda nueva experiencia requirió un proceso de conceptualización que no se redujo a la simple aplicación directa o por analogía de teorías o conceptos preexistentes, pero sí al uso de categorías teóricas para comprender e interpretar el material que emergió. Esta interpretación no se asumió como única, ni como una verdad absoluta, sino como un posible análisis entre otras múltiples miradas. Esta lógica es también consistente con el objeto a estudiar, ya que las reflexiones no son visibles $\mathrm{o}$ asequibles y necesitan ser descritas, analizadas e interpretadas.

La muestra ha sido intencional, los relatos producidos por los residentes fueron seleccionados como punto de partida del trabajo para luego llevar a cabo procesos progresivos y secuenciales de ampliación o reducción de la muestra según las categorías teóricas que fueron emergiendo en el camino, combinado de la obtención y análisis de la información.

En esta investigación, a partir de la teoría que la sustenta y de las recurrencias halladas durante las primeras lecturas de los IC se definieron categorías de análisis que llevaron a una reagrupación y una lectura diferente de los datos. Las lecturas sucesivas nos llevaron a redefinir las categorías, a precisarlas, a distinguir lo que ellas definían. Asimismo, la revisión de la bibliografía relacionada con las categorías seleccionadas y nuevas lecturas de las producciones analizadas favorecieron la reformulación empírica y conceptual.

Para analizar el material recogido se trabajó de la siguiente manera:

- Primera lectura exploratoria de 30 relatos de IC, distribuidos entre los distintos integrantes del equipo de investigación.

- Puesta en común y análisis grupal de las primeras impresiones sobre las lecturas.

- Segunda lectura con el objetivo de detectar tópicos significativos de los IC.

- Análisis grupal de los resultados de la segunda lectura y detección de tópicos recurrentes.

- Entrevistas de ampliación.

Se decidió realizar el análisis comparativo para detectar temas recurrentes en las diferentes producciones de los estudiantes. Se realizaron lecturas selectivas de los tópicos recurrentes y se pusieron en común párrafos textuales para ajustar la selección. Posteriormente se construyeron las siguientes categorías para analizar la muestra completa de documentos seleccionados para esta investigación.

Tabla 1. Categorías construidas para analizar los IC

\begin{tabular}{|c|c|}
\hline $\begin{array}{l}\text { Definición de la } \\
\text { categoría }\end{array}$ & Indicadores \\
\hline $\begin{array}{l}\text { La categoría de relato } \\
\text { refiere a las } \\
\text { características del texto } \\
\text { que desarrollaron los } \\
\text { autores de los IC. }\end{array}$ & $\begin{array}{l}\text { Tipología de textos: } \\
\text { ensanchados y austeros }\end{array}$ \\
\hline $\begin{array}{l}\text { La categoría tareas } \\
\text { organizativas refiere } \\
\text { situaciones evocadas } \\
\text { por los autores de los } \\
\text { IC, que se vinculan al } \\
\text { encuadre y gestión de la } \\
\text { clase. }\end{array}$ & $\begin{array}{l}\text { Fragmentos que hacen } \\
\text { referencia a: } \\
\text { - El sostenimiento de reglas, } \\
\text {-La organización del } \\
\text { espacio. } \\
\text { - La distribución del tiempo } \\
\text {-Al manejo o gestión de la } \\
\text { clase. }\end{array}$ \\
\hline $\begin{array}{l}\text { La categoría tareas de } \\
\text { socialización refiere a } \\
\text { situaciones evocadas } \\
\text { por los autores de los } \\
\text { IC, que se vinculan a } \\
\text { aspectos vinculados a la } \\
\text { construcción de la } \\
\text { posición docente y del } \\
\text { rol profesional y /o a } \\
\text { los vínculos con los } \\
\text { docentes y residentes. }\end{array}$ & $\begin{array}{l}\text { Fragmentos que hacen } \\
\text { referencia a: } \\
\text { - Las concepciones acerca } \\
\text { del rol y la posición docente, } \\
\text { - Los vínculos entre los } \\
\text { docentes y residentes. }\end{array}$ \\
\hline $\begin{array}{l}\text { La categoría de "lo } \\
\text { académico" refiere a } \\
\text { situaciones evocadas } \\
\text { por los autores de los } \\
\text { IC, que se vinculan con } \\
\text { el contenido a enseñar, } \\
\text { la estrategia de } \\
\text { enseñanza y el tipo de } \\
\text { actividades planteadas. }\end{array}$ & $\begin{array}{l}\text { Fragmentos que hacen } \\
\text { referencia a: } \\
\text {-Los contenidos } \\
\text { disciplinares de referencia. } \\
\text {-La estrategia de enseñanza. } \\
\text {-Las relaciones entre los } \\
\text { saberes de los residentes y } \\
\text { los contenidos. } \\
\text {-Tipo de demanda cognitiva } \\
\text { prevista para los residentes. }\end{array}$ \\
\hline
\end{tabular}

Se llevaron a cabo 5 entrevistas en el caso de los relatos muy breves, con el objeto de ampliar la información.

\section{RESULTADOS}

En primer lugar, interesa señalar algunas de las características de los textos escritos. Podemos definir dos grandes grupos: el de los textos ensanchados y el de los textos austeros. Ambos tipos de relatos son pertinentes a la categorización establecida para los IC. Sin embargo, los 
denominados IC ensanchados, son aquellos que reúnen las siguientes características:

- Extensión mayor a 170 palabras.

- El relato describe un suceso vivido, con las características previstas para un IC.

- Lo que lo define como tal es el tipo de relato construido: se describe la situación con gran nivel de detalle, se incluyen preguntas, o reflexiones, aparecen valoraciones o se expresan sentimientos. Es decir, se va más allá de la descripción, se muestran matices que permiten inferir lo inquietante o provocador que resulta ese incidente en el marco de la construcción de una "identidad docente". En algunos relatos, además, se incorporan referencias a aportes teóricos.

Los IC austeros:

- Se extienden hasta 170 palabras.

- El relato describe un suceso vivido, con las características previstas para un IC.

- El relato que se construye es una descripción detallada de la situación vivida. No se incluyen valoraciones o sentimientos, ni preguntas. Tiene el formato de descripción casi objetiva.

Resulta interesante proponer esta diferenciación entre tipos de escritura considerando la potencialidad para la formación docente. Mucha de la literatura especializada otorga valor a la construcción de narraciones como vehículo para la reflexión.

Según Schön (1997), la reflexión es una forma específica de pensar en las prácticas profesionales, un conocimiento en acción que no es meramente verbal. Cuando el profesional reflexivo trata de explicarse lo que pasa, reflexiona también sobre los conocimientos que estaban implícitos en su acción; conocimientos que saca a la luz, critica, reestructura e incorpora a acciones posteriores. Estos conocimientos no se refieren a una reflexión de sus prácticas analizadas en un contexto sociopolítico o cultural sino en el interior del aula para contribuir a su desarrollo profesional.

Estamos en condiciones de afirmar que la escritura de IC habilita la reflexión sobre la enseñanza, más allá de las cualidades o predisposiciones que los "escritores" tengan en relación con el proceso de escritura. Por supuesto, aquellos relatos catalogados como ensanchados, brindan excelentes posibilidades para el análisis (Anijovich y Cappelletti, 2014). Los IC austeros también constituyen una buena fuente de indagación, de formulación de preguntas, para su posterior análisis.
Solo consideraremos en este trabajo los IC referidos al nivel superior debido a los requisitos de extensión para esta publicación.

En relación con los IC referidos al nivel superior nos preguntamos dos cuestiones:

- Si los resultados refieren a que resulta de menor complejidad enseñar Ciencias Jurídicas en el nivel superior.

- Si el tipo de intervención de los estudiantes en este nivel resulta menos provocativa, dado que se refiere por lo general más a los contenidos y no está impactada por características propias del vínculo de la población -en general adolescente- del nivel secundario.

Otro de los aspectos a referir lo constituyen los modos de categorizar los IC: no se trata solo de presentarlos en relación con el nivel de enseñanza en el que se produjeron, sino de hallar lógicas para su tematización. En este sentido, la primera referencia la constituye aquello que Cols (2011) menciona en relación con la esfera de lo organizativo en la enseñanza: “... ligada a la definición y sostén de un encuadre de trabajo y al manejo o gestión de la clase..." (p. 74). Tal como menciona Cols, esta categorización resulta útil desde el punto de vista analítico, pero no puede pensarse en términos de entidades discretas.

“...Un tema no menor que se me planteó en la $2^{\circ}$ clase que di fue el cálculo del tiempo. Si bien en la planificación se habían detallado los tiempos (...) faltaban como 20' para terminar..." (IC n $\left.{ }^{\circ} 8\right)$.

La segunda categoría refiere a lo académico. Los IC problematizan aspectos que involucran contenidos disciplinares de referencia, saberes previos de los residentes, la distancia entre unos y otros de los mencionados anteriormente. También al tipo de estrategia de enseñanza en función del contenido, al tipo de demanda cognitiva prevista para los residentes. Esta categorización toma como referencia la definición de tarea académica propuesta por Doyle (1998), en el sentido que los estudiantes probablemente aprenderán aquello que las actividades que se les proponen los mueva a realizar. Pero, al mismo tiempo, excede a esta apreciación: tal como lo planteamos en este trabajo de investigación, la categoría de lo académico reúne a aquellos relatos que se preguntan, cuestionan o problematizan contenidos, saberes, recursos previstos para las clases.

“...Finalizada la explicación, reparto la actividad, que consistía en que, a través de una nota periodística, determinaran cuál era la cámara de origen y cuál la revisora. A medida que fui pasando por los grupos fui advirtiendo que 
TODOS cometían el mismo error: leían la primera oración del artículo y llegaban a una conclusión errónea. Casi me muero, todos habían entendido mal..." (IC no 11).

La tercera categoría que ponemos en juego es la que denominamos socialización. En torno a ella se agrupan aquellos relatos de IC que ponen de relevancia aspectos muy vinculados a la construcción de la posición docente, a las prácticas profesionales, al ejercicio del rol, a la tensión entre novato y experto o a los vínculos con los docentes y estudiantes. Es aquella que prioriza aspectos de la institucionalización del docente novel, en el sentido de su socialización profesional como docente en el marco de la institución escolar.

“...El profesor a cargo del curso de Práctica Profesional, me adelantó que quería hacer una pequeña introducción. (...) Para mi asombro y estupefacción, lo que sería una pequeña introducción se transformó en una clase compartida donde sus palabras (las del profesor) no me servían de pie para mi clase..." (IC n $\left.{ }^{\circ} 4\right)$.

Los IC brindan la posibilidad de hacer visible algún o algunos de los aspectos que han resultado lo suficientemente potentes como para marcar o dejar huella. Son momentos reconocidos y visibilizados como formativos por el sujeto que los vivenció. El solo hecho de reconocerlos y narrarlos resulta una oportunidad reflexiva. Aun cuando se trate de una descripción anecdótica, ofrece una mirada recursiva sobre algún aspecto complejo acerca de las prácticas de enseñanza y de su contexto.

Como mencionamos anteriormente solo $11 \mathrm{de}$ los 30 IC relevados corresponden al nivel superior. Esto nos lleva a pensar que probablemente la enseñanza universitaria para estos docentes en formación reviste menor complejidad que la enseñanza de nivel secundario, dada la cercanía con el espacio de la propia formación profesional. Consideramos que es posible que adviertan ese carácter complejo una vez que efectivamente comiencen a trabajar como docentes. Es necesario señalar que el marco en el que se lleva a cabo el Profesorado en Ciencias Jurídicas es el de la Facultad de Derecho de la Universidad de Buenos Aires, mismo espacio en el que los docentes en formación realizaron su carrera de Abogacía. Este no es un dato menor: conocen el lugar y están familiarizados con el modo en que se enseña allí. Camilloni (2010) sostiene, al referirse a la enseñanza en el nivel superior, que existen una serie de creencias compartidas por docentes, residentes en general que dar clase en este nivel no reviste mayor complejidad dado que se cree que basta con saber de la asignatura para saber cómo enseñar. La autora sostiene que es necesaria una didáctica científica propia del nivel que se aleje del sentido común, desde la cual los docentes puedan dar sentido a sus prácticas, comprendiendo la complejidad de las mismas y encontrando diversidad de alternativas para pensar sus clases.

Si bien los futuros docentes, a lo largo del trayecto de formación en el profesorado, abordan en profundidad contenidos propios de la didáctica, es posible que algunas ideas ligadas a las representaciones sociales mencionadas anteriormente requieran mayor tiempo de trabajo, que no se limita a la instancia de la formación sistemática brindada por el profesorado. Lo expuesto hasta aquí nos lleva a pensar que probablemente la ausencia de IC referidos a la socialización en este nivel se deba a lo recientemente referido. Los residentes no parecen interrogarse acerca de la construcción del rol en el nivel superior $\mathrm{o}$, por lo menos, no aparece representado en sus producciones.

\section{DISCUSIÓN}

\subsection{Sobre lo académico:}

Del análisis de los IC se obtiene que, en relación con lo académico, se narran situaciones en las cuales los docentes tienen que tomar microdecisiones:

- En un caso se trata de una situación de enseñanza en la que a partir de la presentación de una noticia periodística, se da una discusión que no estaba prevista. Si bien puede resolver la situación en el momento de la clase, la autora plantea que para próximas situaciones será necesario cambiar el recorte de diario presentado, dada la sensibilidad de su contenido, así como acotar los tiempos para ajustarse a los objetivos planteados. Si bien a simple vista, podría pensarse que este IC describe una situación que supone un problema en la gestión del tiempo, en realidad lo que está en juego además de la dimensión temporal es el manejo de la discusión en clase (cómo coordinarlo, darle lugar y cierre) así como la elección apropiada del recurso didáctico.

"...Una de las estudiantes realiza un comentario respecto de la situación de vulnerabilidad en la cual se encuentran muchos menores, y la justificación respecto de dichos hechos. Acto seguido, 
otro le responde con un criterio totalmente diferente y en ese momento comenzaron a hablar todos al mismo tiempo y a elevar el tono de voz. Les pedí que continuáramos con la clase, seguí con el tema desarrollado, escribí las características en el pizarrón para que fijaran su atención en ello y por último, antes de cerrar la clase les dejé como consigna, qué tema les parecía que podíamos seleccionar para organizar un debate, de acuerdo con los contenidos de la materia que ellos tenían..." (IC n 25).

Un residente describe una situación en la que, a partir de una exposición dialogada, los residentes demostraban no comprender lo que el practicante intentaba explicar. Este último comenta que el contenido a transmitir tomaba distintas categorizaciones y que, al escuchar su exposición, los estudiantes expresaban en sus caras no comprender. Esto lo llevó a leer textualmente sus notas. En su relato este residente da cuenta de una reflexión crítica dado que busca alternativas diferentes para resolver esta situación (usar el pizarrón, organizar de otro modo los contenidos) y apela a los saberes teóricos del campo didáctico para pensar en la situación vivida. Esto lo lleva a revisar la tensión entre extensión y profundidad.

“...Mientras transmitía, los estudiantes me empezaron a hacer preguntas que les contestaba, por otro lado, estaba la presentación de la clasificación, las características del tipo de remuneración y (...) veía caras atónitas y ceños fruncidos, recuerdo que en ese momento me corrió una gran tensión porque pensaba en los propósitos y objetivos de mi clase y hasta dónde había llegado el alcance de estos..." $\left(\mathrm{IC} \mathrm{n}^{\circ} 7\right)$.

\subsection{Sobre lo organizativo}

Respecto de lo organizativo, los incidentes refieren a:

- El manejo del tiempo. En uno de los relatos aparece claramente cómo la variable tiempo tiene un peso importante en las microdecisiones de los residentes. La residente plantea que por haber empezado la clase treinta minutos después del horario previsto tuvo que reformular su planificación para llegar a enseñar los contenidos planificados. Es interesante en este caso cómo un incidente de tipo organizativo conlleva una decisión de tipo académica. La autora señala que, al detectar la falta de tiempo, decidió en el momento abordar problemas de la vida cotidiana y dejar de lado la actividad que había planificado. Además del relato se desprende una reflexión dialógica en la que menciona las preguntas y comentarios que fue pensando mientras llevaba adelante la clase.

“...La 2da clase comenzó 30 'más tarde de lo esperado, ahí surgió el primer incidente crítico, el factor determinante 'el tiempo' "...pensé no es conveniente que realicen la actividad en grupos de 'historia clínica' les va a demandar más de $20^{\prime}$ o más (...) Decidí comenzar con un breve repaso de los temas anteriores para saber adónde estaba parada..." (IC n $\left.{ }^{\circ} 23\right)$.

- Otro relato da cuenta del uso inesperado de un recurso que no había sido planificado. El autor explica que le sobró tiempo en su clase, que no sabía qué hacer y entonces tomó un texto para compartir con sus estudiantes, que generó gran interés. El practicante da cuenta de cómo el uso de un recurso que no había sido pensado para esa clase habilitó un espacio de intercambio.

“...Un tema no menor que se me planteó en la $2^{\circ}$ clase que di fue el cálculo del tiempo. Si bien en la planificación se habían detallado los tiempos (...) faltaban como 20' para terminar (...) Saqué un artículo que leí como antecedente para preparar mis clases. Resultó un recurso interesante (...) ese texto no formaba parte de mi próxima exposición. Resultó oportuno y motivó a los residentes (...) fue un cierre con mucha intervención y que generó mucho interés y dejó abierto el tema para comenzar a trabajarlo la próxima clase.... (IC $\left.\mathrm{n}^{\circ} 8\right)$.

- El funcionamiento incorrecto de un recurso. Una residente narra que al momento de dar su práctica no funcionaron adecuadamente los parlantes que darían sonido a un fragmento de película. Si bien pudo resolver el problema técnico adecuadamente, lo vivió con nervios dado lo inesperado de la situación.

“...Para la clase había planificado que trabajaran sobre un documental, y había 
formulado distintas actividades sobre eso. Pero el proyector de la institución no tenía parlantes y yo no había previsto esto. Realmente se escuchaba bajísimo. Me puse muy nerviosa. Tuve que salir afuera del aula a pedirle a los residentes que estaban charlando en el pasillo que por favor hicieran silencio. Y les pedí a los alumnos que se juntaran más..." (IC n ${ }^{\circ} 2$ ).

\subsection{Sobre la socialización}

Respecto de este aspecto, solamente se presenta un incidente en este nivel. Sin embargo, es interesante considerar lo que sucede dado que es paradigmático de este tipo de IC. Se refiere a:

- El vínculo y articulación con el docente a cargo del curso. Una residente relata una situación en la que minutos antes de ingresar al aula el docente titular decidió cambiar los contenidos de la clase y compartir el "dictado" de esa clase con ella. El relato da cuenta de cierto grado de competencia entre el titular y la residente por quién se hace cargo de la clase y por aquello a ser enseñado. Es interesante cómo ese vínculo, en el que se pone en juego la autoridad y el reconocimiento, puede (re)configurar una situación de enseñanza especialmente cuando se trata de una práctica docente.

“...El profesor a cargo del curso de Práctica Profesional, me adelantó que quería hacer una pequeña introducción. (...) Para mi asombro y estupefacción, lo que sería una pequeña introducción se transformó en una clase compartida donde sus palabras (las del profesor) no me servían de pie para mi clase (...)"entonces resolví tomar impulso e imponerme. Tenía la clase muy preparada y una secuencia de contenidos y actividades muy trabajadas con varias herramientas. Creo que a pesar de su gran histrionismo logré que se corriera del centro de la clase, y que se mantuviera al margen, traté de tenerlo como aliado..." (IC n $\left.{ }^{\circ} 4\right)$.

El análisis de los IC estudiados nos permite advertir que los residentes parecen preocuparse más por cuestiones organizativas que por aspectos ligados a la dimensión académica. Los ruidos, los cambios de último momento, el control del tiempo, etc. parecen ser factores que desestabilizan y/o preocupan al residente. Asimismo, en la mayoría de las producciones se hace mención a la necesidad de hacer cambios en la programación de la clase. En ese sentido, si bien la referencia inicial es a cuestiones organizativas, estas devienen en decisiones didácticas ligadas a la articulación entre lo programado y lo ejecutado. Pareciera que la programación juega un papel importante para estos residentes y las microdecisiones refieren a la distancia o cercanía que pueden sostener entre lo planificado y lo que sucede en el devenir de la clase.

Respecto a las cuestiones académicas mencionadas en los IC aparecen aspectos ligados a alcanzar la comprensión de los estudiantes, al manejo de la discusión en el aula y al uso adecuado de los recursos para generar discusión y participación.

\section{CONCLUSIONES}

Deseamos hacer referencia a las categorías construidas para el análisis. Tal como mencionamos, los IC refieren a tres cuestiones: o bien a lo académico, o bien a lo organizativo de la clase o bien a cuestiones de socialización profesional. Esto resulta coherente con los aspectos que se identifican como problemáticos en la formación docente en relación con la planificación.

El contenido que se pone de manifiesto en los IC, puede pensarse teniendo en cuenta la condición de docente novato/docente en formación, de los residentes. Esto impactaría tanto en los IC que refieren a cuestiones académicas como a aquellos que refieren a aspectos organizativos. Ambos presentan preocupaciones centrales de toda propuesta de clase.

Según Perrenoud (2004, p. 11)

...Una situación de clase presenta en general múltiples componentes, que hay que tratar de forma coordinada, incluso simultánea, para llegar a una acción acertada. El profesional dirige la situación globalmente, pero moviliza ciertas competencias específicas, independientes las unas de las otras, para tratar ciertos aspectos del problema, al modo de una empresa que subcontrata algunas operaciones de producción. Sabemos, por ejemplo, que los profesores experimentados han desarrollado una competencia muy preciada: la de percibir simultáneamente múltiples procesos que se desarrollan a la vez en su clase. El profesor experto «tiene ojos en la espalda», es capaz de advertir lo esencial de lo que se trama en varias escenas paralelas, sin que 
ninguna lo deje estupefacto o lo angustie...

Las situaciones referidas muestran que esta metáfora de "ojos en la espalda" se encuentra lejos de ser alcanzada. Sin embargo, la documentación y análisis de IC pueden conformar un dispositivo valioso para su construcción.

Destacamos la potencia del trabajo con dispositivos narrativos para la formación de futuros profesores a través de las siguientes ideas:

- Propician que sus autores reflexionen porque el solo hecho de narrar implica reconstruir y revisar lo sucedido.

- Dado que se trata de sucesos identificados como complejos para quien los relata, que lo ha conmovido de algún modo, el registro permite conservar impactos emocionales. Además, la evocación del incidente promueve la emergencia de huellas potentes en sus procesos formativos fuera del trayecto formal de formación.

- Habitúan a sus autores a escribir y documentar sus prácticas.

- Proporcionan una retroalimentación inmediata y permanente al autor, que se convierte en lector de su propia experiencia.

- La narración escrita de situaciones vividas permite compartir experiencias con pares $\mathrm{y}$ docentes.

Podemos señalar que los IC ofrecen oportunidades valiosas para el trabajo sobre las diferencias entre las teorías implícitas y las adoptadas (Argyris y Schon 1974). En ellos se pone en evidencia la distancia entre lo planificado en la instancia preactiva y las posibilidades reales de acción. Los IC expresan preocupaciones concretas, reales, situaciones conflictivas y modos de resolución, que pueden resolverse por acción tomando alguna definición, realizando algo-, o por omisión: no se hace nada con la situación en ese momento, pero se identifica y se revisa en un momento posterior, marcando una diferencia prospectiva.

Puede asegurarse que la narración y la escritura de IC ofrecen descripciones, reflexiones, datos, narraciones sobre hechos, hipótesis, anticipaciones y conexiones entre teoría y práctica. De este modo, constituyen un material rico y variado para el análisis e interpretación.

A continuación, mencionamos cinco nudos de significados que construimos a partir de esta investigación.
1. Los sucesos que impactan y construyen subjetividad en los docentes noveles contienen preocupaciones por el contenido a enseñar. En términos de pensar el trayecto de formación docente, este aspecto podría ser central a la hora de promover análisis de situaciones de enseñanza, elaborar estrategias, formular planificaciones.

2. Las reflexiones acerca de los problemas con la construcción del contenido escolar son advertidas en su mayor parte a partir de la escucha atenta de los docentes en sus clases: el análisis de las respuestas de los estudiantes, o sus preguntas, el tipo de uso realizado de algún recurso previsto permite reflexionar sobre aspectos no previstos o considerados al momento de planificar las clases.

3. En la formación de docentes, esto puede constituir un insumo valioso, donde se invite a focalizar en las intervenciones de los estudiantes considerando qué es lo que ellas desvelan para poder mejorar la enseñanza.

4. Las reformulaciones que las clases sufren en relación con variables no necesariamente anticipadas por los docentes noveles. Estas reformulaciones son producto de sus decisiones o microdecisiones, que cambian el rumbo de lo planificado en función de sostener los propósitos de la enseñanza o bien de reorientarlos. Este aspecto resulta central para la formación pedagógica, dado que permite interrogar la función de la planificación en la enseñanza, y al paradigma sobre el cual se realiza. Permite también focalizar en la formación de una de las competencias generales más valorada: la flexibilidad para poder adaptarse a las nuevas situaciones sosteniendo los propósitos de los cuales se parte.

5. El peso otorgado a situaciones en las cuales se percibe como diluida la propia profesionalidad docente o se ve interpelada por las intervenciones de otros docentes: situaciones donde "no se sabe bien qué hacer, pero se hace igual", tratando de fijar posición.

$\mathrm{Si}$ bien al análisis externo estas situaciones pueden parecer superficiales para ser tratadas en el marco de la formación docente, la potencialidad 
para el análisis puede ser de valiosa: se trata de la construcción del perfil profesional. En este caso, el análisis de los IC puede visibilizar sentidos construidos acerca de la profesionalidad docente que resulte valioso deconstruir o según se trate, sostener.

En otro sentido, otro análisis que puede realizarse es acerca del tipo de conocimiento que deviene de los relatos de los IC. Las narraciones están centradas en lo episódico y lo anecdótico, que es propio del dispositivo. Se trata de vivencias de cada uno de los que se están formando, vivencias "de vida" que se solicita que evoquen. Pero al mismo tiempo son situaciones que generan reflexión. El IC está signado por la práctica y al mismo tiempo, permite pensar en el futuro de esas prácticas, y los modos de confirmarlas $\mathrm{o}$ revisitarlas modificadas.

Los IC pueden entenderse como experiencias subjetivas situadas. Los conocimientos situados pueden entenderse en términos de "visiones" en las que la posición desde la cual se "mira" define las posibilidades de lectura y acción. Es decir, permite posicionamientos en que sólo algunas verdades son posibles. Gracias a esta posición se pueden establecer conexiones parciales con otros agentes para construir conocimiento. Conexiones porque hay lenguajes $\mathrm{y}$ experiencias compartidas $\mathrm{y}$ parciales porque todas las posiciones difieren entre sí y no se conectan a partir de su identidad sino de la tensión entre semejanza y diferencia entre ellas. Es a partir de narrar, de construir los relatos, que resulta posible reflexionar sobre el posicionamiento desde el que se toman decisiones sobre la enseñanza, sobre los contenidos, sobre las estrategias, sobre la relación con los residentes.

Este dispositivo produce una "toma de conciencia" acerca de los obstáculos y de los aprendizajes construidos, y tiene la potencia de iluminar respecto de la construcción del futuro rol como docente.

En términos del trayecto de formación docente la irrupción de nudos que implican las valoraciones, la afectividad, las cuestiones personales, y, a la vez, el trabajo sobre ellas, es evidencia de la articulación entre estas mismas cuestiones personales y las características más instrumentales de las prácticas de enseñanza. Este hallazgo nos permite decir, con la fuerza empírica que brinda la sistematización realizada, que resulta valioso considerar en la formación docente no solamente aspectos teóricos, referencias técnicas, sino también brindar la posibilidad de referirse a los procesos reflexivos sobre "si mismos".
Consideramos que tanto el relevamiento como la información que surja del análisis conforman marcos valiosos para formular estrategias de enseñanza en el marco de la formación de docentes: documentar qué piensan acerca de la enseñanza, cómo lo piensan, cómo se concreta esto en la formulación de actividades, qué ideas surgen acerca de la definición del contenido y de su alcance son algunas de las potencialidades que podrían ser tenidas en cuenta en futuras investigaciones.

\section{REFERENCIAS}

Anijovich, R. et al (2009). Transitar la formación pedagógica. Buenos Aires, Argentina: Paidós.

Anijovich, R, y Cappelletti, G (Comp). La práctica como eje de la formación. Buenos Aires: Eudeba.

Argyris, C., y Schön, D. (1974). Theory in practice: Increasing professional effectiveness. San Francisco, Estados Unidos: Jossey Bass.

Camilloni, A. (2010). La formación de profesionales universitarios. Gestión universitaria. Revista Electrónica de la Universidad Nacional de La Matanza, 2(2).

Cols, E. (2011). Estilos de enseñanza. Rosario, Argentina: Homo Sapiens.

Doyle (1998). Didáctica II N 2. Aportes teóricos. Buenos Aires: Universidad de Buenos Aires.

Fernández González, J., Elórtegui Escartín, N. y Medina Pérez, M. (2003). Los incidentes críticos en la formación y perfeccionamiento del profesorado de secundaria de ciencias naturales. Revista Interuniversitaria de Formación de Profesorado, 17(1), 101-112.

Fernández González, J., Medina Pérez, M., y Elórtegui Escartín, N. (2003). Enseñar a profesores de secundaria con situaciones problemáticas. Revista electrónica de Enseñanza de las Ciencias, 2(3), 263270.

Flanagan, B. (1954). The Critical Incident Technique. Psychological Bulletin, 51(4), 1-33. Recuperado

http://www.apa.org/pubs/databases/psycinfo/citarticle.pdf

Griffin, M.L. (2003). Using Critical Incidents to Promote and Assess Reflective Thinking in Preservice Teachers. Reflective Practice, 4(2), 207-220 DOI: https://doi.org/10.1080/14623940308274.

Husu, J., Toom, A., y Patrikainen, S. (2008). Guided reflection as a mean to demonstrate and develop student teachers reflective competencies. Reflective Practice. 9(1), 37-51.

Harrison J., y Lee, R. (2011). Exploring the use of critical incident analysis and the professional learning conversation in an initial teacher education programes. UK University of Leicester.

Litwin, E. (2008) El Oficio de enseñar, Condiciones y contextos. Buenos Aires, Argentina: Paidós.

Perrenoud, P. (2004) Desarrollar la práctica reflexiva en el oficio de enseñar. Barcelona, España: Grao.

Rosales, C. (2000). Evaluar es reflexionar sobre la enseñanza. Madrid, España: Narcea. 
Schön, D. (1997). La formación de profesionales reflexivos, Barcelona, España: Paidós MEC.

Shulman, J. H. (2002). Happy Accidents: Cases as opportunities for Teacher Learning. San Francisco, West Ed. Recuperado de https://www.wested.org/online_pubs/happyaccidents.p df

Tripp, D. (1993). Critical Incidents in Teaching: Developing Professional Judgement. London, Reino Unido: Routledge. 